\title{
Delivering Enhanced Testosterone Replacement Therapy through Nanochannels
}

\author{
Silvia Ferrati, Eugenia Nicolov, Shyam Bansal, Erika Zabre, Thomas Geninatti, \\ Arturas Ziemys, Lee Hudson, Mauro Ferrari, Randal Goodall, Mohit Khera, \\ Ganesh Palapattu, * and Alessandro Grattoni*
}

Primary or secondary hypogonadism results in a range of signs and symptoms that compromise quality of life and requires life-long testosterone replacement therapy. In this study, an implantable nanochannel system is investigated as an alternative delivery strategy for the long-term sustained and constant release of testosterone. In vitro release tests are performed using a dissolution set up, with testosterone and testosterone:2-hydroxypropyl$\beta$-cyclodextrin (TES:HPCD) 1:1 and 1:2 molar ratio complexes release from the implantable nanochannel system and quantify by HPLC. 1:2 TES:HPCD complex stably achieve 10-15 times higher testosterone solubility with 25-30 times higher in vitro release. Bioactivity of delivered testosterone is verified by LNCaP/LUC cell luminescence. In vivo evaluation of testosterone, luteinizing hormone (LH), and follicle stimulating hormone (FSH) levels by liquid chromatography mass spectrometry (LC/MS) and multiplex assay is performed in castrated Sprague-Dawley rats over $30 \mathrm{~d}$. Animals are treated with the nanochannel implants or degradable testosterone pellets. The 1:2 TES:HPCD nanochannel implant exhibits sustained and clinically relevant in vivo release kinetics and attains physiologically stable plasma levels of testosterone, $\mathrm{LH}$, and $\mathrm{FSH}$. In conclusion, it is demonstrated that by providing long-term steady release 1:2 TES:HPCD nanochannel implants may represent a major breakthrough for the treatment of male hypogonadism.

\section{Introduction}

Incredible progress has been achieved in testosterone replacement therapy (TRT) over the last 70 years. What started as liver-toxic oral formulations, scrotal patches, and multi-daily injections have evolved into topical gel formulations, subcutaneous pellets, and depot injections that can deliver testosterone for days to months. ${ }^{[1]}$ Despite these advances, current delivery approaches are prone to generate significant, and often inconsistent, fluctuations in serum testosterone concentration, a phenomenon that considerably lessens efficacy and may give rise to side effects. ${ }^{[2]}$ Long-lasting injectables or implantable polymeric formulations, the current delivery options with the longest duration of action, are commonly associated with initial peaks in testosterone plasma concentration after implantation followed by a gradual decrease in circulating hormone levels. Further, implantable polymeric approaches present significantly reduced hormone release rates after $\approx 65 \%$ of their payload has been expended. Lastly, these types of delivery methods require frequent re-dosing, every few weeks to months, making long-term therapy cumbersome.

TRT is used to treat hypogonadism defined by testosterone plasma levels of less than $8 \mathrm{nmol}\left(<300 \mathrm{ng} \mathrm{dL}^{-1}\right)$ with accompanying signs and symptoms, ${ }^{[3]}$ such as loss of lean muscle mass, erectile dysfunction, loss of libido, weight gain, infertility, depressed mood, and osteoporosis. ${ }^{[4]}$ A delivery system capable of providing constant,

\footnotetext{
Dr. S. Ferrati, Dr. E. Nicolov, Dr. S. Bansal, E. Zabre, T. Geninatti, Dr. A. Ziemys, Dr. M. Ferrari, Dr. A. Grattoni

The Department of Nanomedicine

Houston Methodist Research Institute

6670 Bertner Avenue, Houston, TX 77030, USA

E-mail: agrattoni@houstonmethodist.org

Dr. S. Bansal

Division of Cardiovascular Diseases The University

of Alabama at Birmingham

$15303^{\text {rd }}$ Avenue South, Birmingham, AL 35294, USA

Dr. L. Hudson, Dr. R. Goodall

NanoMedical Systems Inc.

Austin, TX, 4401 Freidrich Ln 307, Austin, TX 78744, USA
}

\author{
Dr. M. Khera \\ Department of Urology \\ Baylor College of Medicine \\ 7200 Cambridge Street, Houston, TX 77030, USA \\ Dr. G. Palapattu \\ Department of Urology \\ University of Michigan \\ 1500 E Medical Center Drive SPC 5913, Ann Arbor, MI 48109, USA \\ E-mail: gpalapat@med.umich.edu
}

DOI: 10.1002/adhm.201400348 
long-term, and sustained testosterone replacement would notably improve the treatment options for post-pubertal males suffering from androgen deficiency.

We developed an implantable nanochannel system for the constant and sustained delivery of therapeutics. ${ }^{[5-7]}$ The system leverages a silicon membrane presenting geometrically defined nanochannels as small as $2.5 \mathrm{~nm}$ to control the rate of drug release by physicoelectrostatic confinement. ${ }^{[8]}$ The membrane is assembled into an implantable polyethyl-ether-ketone (PEEK) or titanium reservoir. The constant release is achieved by rational selection of the nanochannel size to the drug molecule to allow a steady diffusion of molecules per unit time. By altering the number of nanochannels, through a straightforward scaling process, the device can be customized to vary the dose according to individual patient's needs. We hypothesize that with the implantable nanochannel system, constant and sustained plasma levels of testosterone can be achieved long term.

In this study, we present our system optimized for the release of testosterone. We tested testosterone delivery from membranes presenting four different nanochannel sizes, achieving sub-optimal release rates due to poor testosterone water solubility. To overcome the limitation of low solubility, we formulated inclusion testosterone-2-hydroxypropyl- $\beta$-cyclodextrin (TES:HPCD) complexes, in a $1: 1$ and $1: 2$ molar ratios. The sustained release, stability, and bioactivity of TES:HPCD complexes were tested in vitro, over $30 \mathrm{~d}$, from 5 and $13 \mathrm{~nm}$ nanochannel membranes. Based on the in vitro results, $5 \mathrm{~nm}$ membranes were selected for in vivo testing, assembled into titanium implants loaded with 1:2 TES:HPCD and subcutaneously implanted into castrated Sprague-Dawley rats. Plasma testosterone concentrations were measured by LC/MS and age matched non-castrated male rats were used as physiological controls. In vivo release rates were measured from the residual testosterone in the recovered implants. To verify testosterone bioactivity in vivo, we assessed plasma levels of luteinizing hormone (LH) and follicle stimulating hormone (FSH).

\section{Results}

\subsection{In Vitro Testosterone Release and Bioactivity}

To determine the appropriate nanochannel size for constant release of testosterone (Figure 1a), we measured testosterone release in vitro from 3, 13, 20, and $1000 \mathrm{~nm}$ membranes (Figure 1b,c). Notably, all nanochannel sizes displayed sustained release kinetics, with an average release rate of $18-20 \mu \mathrm{g} \mathrm{d}^{-1}$.

A critical challenge in long-term delivery of endocrine hormones is maintaining biological efficacy. To test this, bioactivity of released testosterone was examined using LNCaP cells stably transfected with the luciferase gene under the control of an androgen-dependent promoter. Released testosterone induced luminescence of LNCaP cells (Table 1). Testosterone concentrations measured by LNCaP luminescence assay (concentration of bioactive testosterone) were found to be 1.2-1.4 times higher than determined by HPLC (actual testosterone concentration), likely indicating the greater sensitivity and inherent variability of cell culture.

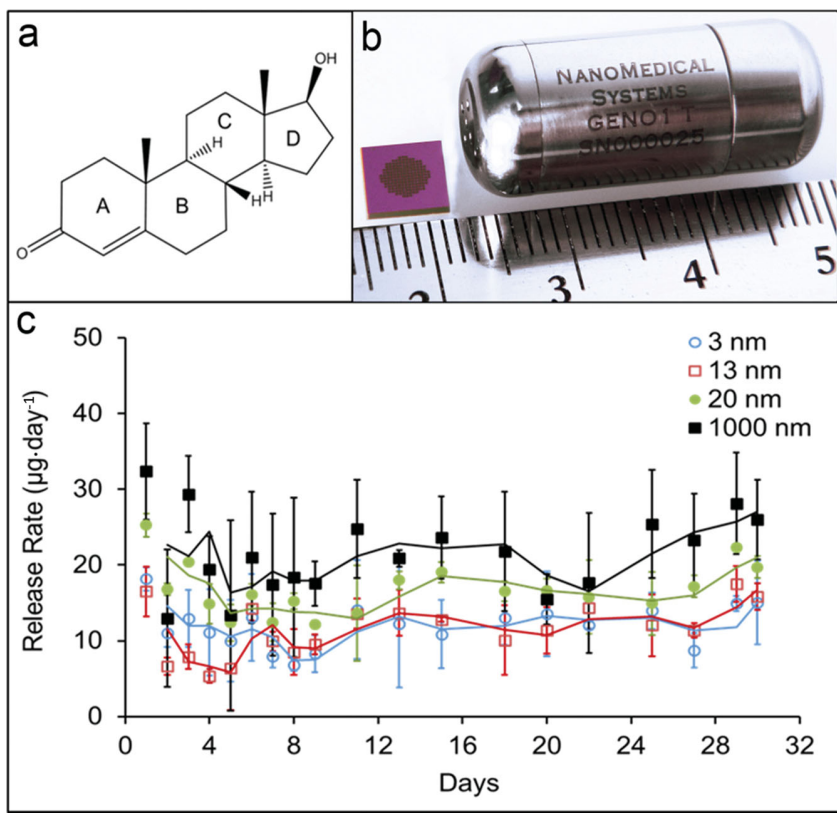

Figure 1. In vitro release. a) Chemical structure of testosterone. b) nanochannel membrane next to the assembled implant. c) Average daily in vitro release of testosterone from 3, 13, 20, and $1000 \mathrm{~nm}$ nanochannel membranes. Results shown are an average of $n=3$ for each nanochannel size except for $1000 \mathrm{~nm}$ where only two replicates were used.

\subsection{Characterization and Optimization of TES:HPCD Inclusion Complexes}

Given a testosterone release rate of $18-20 \mu \mathrm{g} \mathrm{d} \mathrm{d}^{-1}$ (Figure 1) would not be therapeutically sufficient, we sought to alter the testosterone formulation to improve release rates. For this, we formulated water-soluble inclusion complexes of testosterone with HPCD macrocyclic oligosaccharide. A qualitative analysis of TES-HPCD interactions based on enthalpy and entropy energy analysis was performed. Graphical representation of 1:1 and 1:2 TES:HPCD complexes is shown in Figure 2a. The 1:2 molar ratio TES:HPCD originate lower energy interactions suggesting the formation of a more stable complex (Figure $2 \mathrm{~b}$ ). The phase solubility study demonstrated that testosterone solubility increased proportionately at increasing HPCD concentrations reaching a plateau at $2 \times 10^{-3} \mathrm{M}$ (Figure $2 \mathrm{c}$ ). We determined the

Table 1. Comparison of testosterone concentration measured by HPLC and proliferation assay using LNCaP cells $(n=6)$ as compared to a negative control.

\begin{tabular}{lcccc}
\hline $\begin{array}{l}\text { NanoCH. Size } \\
{[\mathrm{nm}]}\end{array}$ & \multicolumn{2}{c}{$\begin{array}{c}\text { TES concentration } \\
{\left[\times 10^{-9} \mathrm{M}\right]}\end{array}$} & & $\begin{array}{c}\text { Conc. } \\
\text { ratio }\end{array}$ \\
\cline { 2 - 4 } & LnCaP Cells & HPLC & LnCaP/HPLC \\
\hline 3 & $3.4 \pm 0.4$ & $2.6 \pm 0.4$ & 1.31 \\
13 & $3.8 \pm 1.1$ & $2.6 \pm 0.3$ & 1.46 \\
20 & $3.3 \pm 0.1$ & $2.6 \pm 0.1$ & 1.27 \\
1000 & $3.3^{\mathrm{a})}$ & 2.7 & 1.22 \\
Negative control & $0.03 \pm 0.008$ & 0.00 & & - \\
\hline
\end{tabular}

a) SD is not shown as only two replicates were used. 


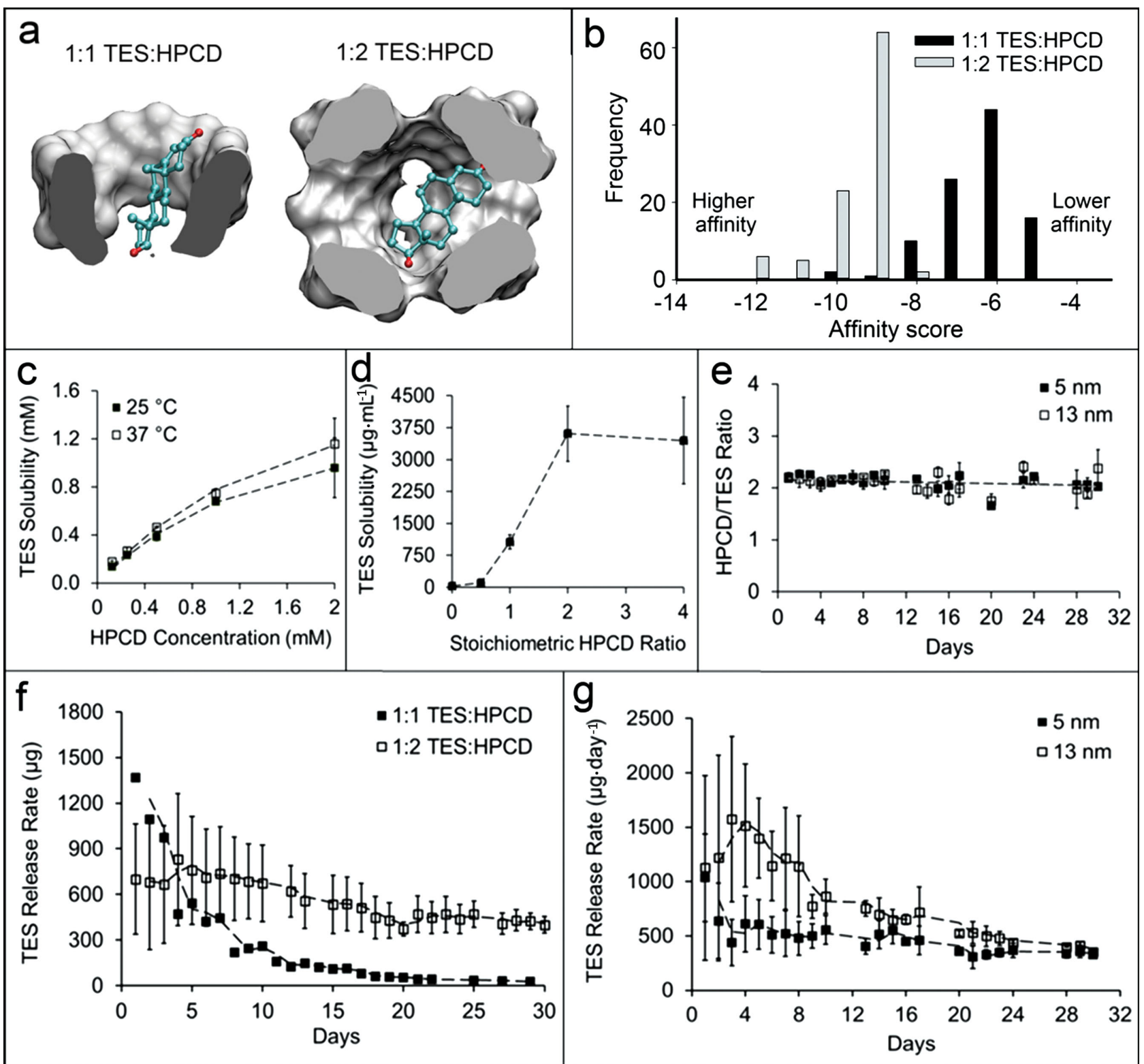

Figure 2. Testosterone-cyclodextrin complex. a) Graphical representation of 1:1 and 1:2 TES:HPCD complexes. b) Affinity score for 1:1 and 1:2 TES:HPCD complexes based on enthalpic and entropic analysis. c) Testosterone solubility enhancement with increasing concentrations of HPCD. d) Solubility of testosterone inclusion complexes prepared with 1:0.4, 1:1, 1:2, and 1:4 stoichiometric ratio of testosterone to HPCD. e) Molar ratio of HPCD to testosterone released from 5 and $13 \mathrm{~nm}$ membranes. f) Average daily release profile of testosterone inclusion complexes with 1:1 and 1:2 stoichiometric ratios of testosterone to HPCD, from $5 \mathrm{~nm}$ nanochannels. g) Average daily release profile of 1:2 TES:HPCD inclusion complexes from 5 and $13 \mathrm{~nm}$ nanochannels.

stability constants for complexation (see Supporting Information Section 2) and found them to decrease from $3212 \mathrm{M}^{-1}$ at $25{ }^{\circ} \mathrm{C}$ to $2057 \mathrm{M}^{-1}$ at $37{ }^{\circ} \mathrm{C}$. At plateau, testosterone solubility was $1 \times 10^{-3} \mathrm{M}$, exhibiting a stoichiometric ratio of 1:2 between testosterone and HPCD (Figure 2c). We prepared inclusion complexes of testosterone using different HPCD concentrations (1:0.4, 1:1, 1:2, and 1:4 molar ratios) and tested them for solubility enhancement. Testosterone solubility increased rapidly as the molar ratio of HPCD increased from 0.4 to 1 and maximum testosterone solubility $\left(3610 \pm 651 \mu \mathrm{g} \mathrm{mL} \mathrm{mL}^{-1}\right)$ was achieved at a molar ratio of 1:2 (TES:HPCD) (Figure 2d).

\subsection{In Vitro Release Kinetics of Testosterone Inclusion Complexes}

To examine if TES:HPCD complex could be delivered through nanochannels and maintain stability, we examined the release kinetics of $1: 1$ and 1:2 inclusion complexes from $13 \mathrm{~nm}$ nanochannel system. The concentration of TES and HPCD in the samples determined by HPLC and a clororimetric assay, respectively, showed that the TES-HPCD ratio in the complex was maintained after being released through nanochannels. At 1:1 stoichiometric ratio, the release rate decreased substantially 
over $30 \mathrm{~d}$ (Figure $2 \mathrm{f}$ ), reaching values of $25-30 \mu \mathrm{g} \mathrm{d}^{-1}$ after $20 \mathrm{~d}$, comparable to the release of testosterone alone. The delivery rate obtained with 1:2 TES:HPCD solution demonstrated sustained release kinetics at $557 \pm 180 \mu \mathrm{g} \mathrm{d}^{-1}$ for $30 \mathrm{~d}$ (Figure 2f). The 1:2 TES:HPCD ratio is therefore ideal to generate constant testosterone release from implantable nanochannel system.

\subsection{Effect of Nanochannel Size on the Release of Testosterone Inclusion Complex}

To determine the appropriate nanochannel size for long-term TRT, we compared the release of 1:2 TES:HPCD from 5 and $13 \mathrm{~nm}$ nanochannel system. We have previously shown that altering nanochannel size can influence release rates. ${ }^{[7,14]}$ As shown in Figure 2e, the profile from $13 \mathrm{~nm}$ nanochannels gives an initial burst release of $1573 \pm 760 \mu \mathrm{g} \mathrm{d}^{-1}$ that decreases to $864 \pm 159 \mu \mathrm{g} \mathrm{d}^{-1}$ by day 10 . In contrast, $5 \mathrm{~nm}$ nanochannels exhibited a relatively constant release of $478 \pm 158 \mu \mathrm{g} \mathrm{d}^{-1}$ (Figure 2e), likely due to the increased confinement on diffusing molecules. To determine the stability of the complex, we measured HPCD concentration in released samples. A constant TES/HPCD molar ratio of 1:2.1-1:2.2 was observed over $30 \mathrm{~d}$ (Figure 2c) demonstrating that the 1:2 complex is stable and is released intact.

\subsection{Plasma Testosterone Concentration}

To evaluate our implantable nanochannel system as a possible TRT strategy, titanium implants (Figure 1b) with $5 \mathrm{~nm}$ membranes were loaded with 1:2 TES-HPCD and subcutaneously implanted into 7-8-month-old male castrated Sprague-Dawley rats. We measured plasma testosterone concentrations by LCMS and age matched non-castrated male rats were used as physiological controls. Implants with PBS or HPCD alone, resulted in comparable $(p=0.32)$ and 10 times lower plasma testosterone levels as compared to non-castrated physiological controls (Figure 3a). Implants with testosterone powder produced increased plasma testosterone levels $\left(0.75 \pm 0.39 \mathrm{ng} \mathrm{mL} \mathrm{m}^{-1}\right)$ as compared to PBS loaded implants $\left(0.31 \pm 0.14 \mathrm{ng} \mathrm{mL}{ }^{-1}\right)$ but the levels remained lower than the non-castrated controls $\left(1.66 \pm 0.46 \mathrm{ng} \mathrm{mL}^{-1}\right)$. In contrast, TES:HPCD implants achieved and maintained plasma testosterone concentrations $\left(2.58 \pm 1.53 \mathrm{ng} \mathrm{mL} \mathrm{m}^{-1}\right)$ comparable to physiological controls $(p=0.30)$. As a positive control of testosterone replacement, one half of a degradable polymeric pellet was implanted in castrated rats and exhibited biphasic release kinetics (Figure 3a and Supporting Information).

To verify the in vivo release rate, we measured the residual testosterone from recovered implants. We determined that TES:HPCD implants had a fourfold higher in vivo release rate compared to testosterone powder loaded implants $(620 \pm$ $50 \mu \mathrm{g} \mathrm{d}^{-1}$ vs $\left.152 \pm 20 \mu \mathrm{g} \mathrm{d}^{-1}, p=0.02\right)$. Interestingly, the in vivo release of powder testosterone was 8-10 times higher than the in vitro release rate (Figure 1c).

\subsection{Plasma $\mathrm{LH}$ and $\mathrm{FSH}$}

To confirm the physiological function of implant-released testosterone, we assessed plasma levels of LH and follicle stimulating hormone (FSH). ${ }^{[3,15]}$ Baseline plasma levels of $\mathrm{LH}$ and FSH in castrated rats were several folds higher than noncastrated controls (Figure 3b,c). Castrated animals with either PBS or testosterone powder nanochannel implants also displayed significantly elevated levels of LH $\left(28-30 \mathrm{ng} \mathrm{mL} \mathrm{m}^{-1}\right)$ and FSH (160-180 ng $\left.\mathrm{mL}^{-1}\right)$. On day 7, animals with TES:HPCD implant similarly exhibited elevated levels of LH and FSH but the levels were lower than PBS implant group (19.8 \pm 12.4 and $\left.122 \pm 54 \mathrm{ng} \mathrm{mL} \mathrm{m}^{-1}\right)$. At day 21 and 35 , LH levels were found to be in the physiological range $(p=0.4)$ but FSH levels remained high (Figure $3 \mathrm{~b}, \mathrm{c})(p=0.0008)$. It is to be noted that some castrated rats had $\mathrm{LH}$ and FSH beyond the range of the standard curve, hence, they were considered to be at the highest standard concentration (30 and $300 \mathrm{ng} \mathrm{mL} \mathrm{m}^{-1}$, respectively). With degradable pellet, $\mathrm{LH}$ levels were below the physiological range $(0.13 \pm$ 0.05 and $0.16 \pm 0.13 \mathrm{ng} \mathrm{mL} \mathrm{m}^{-1}$ on day 21 and $\left.35(p=0.03)\right)$.

\section{Discussion}

Hypogonadism and related sequelae pose a significant health burden on men. This issue is only expected to increase as the

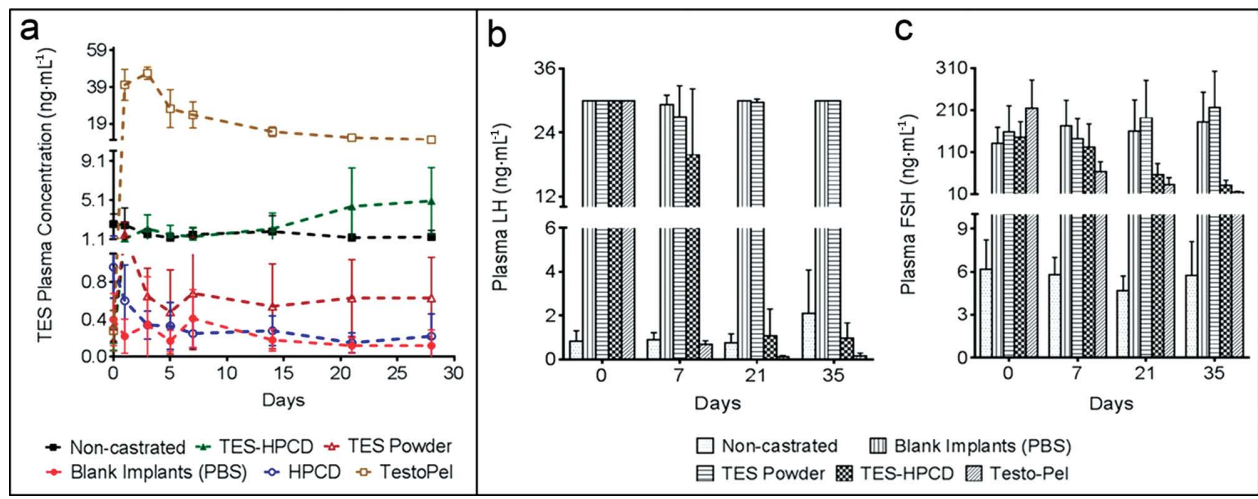

Figure 3. In vivo release. a) Plasma testosterone concentrations obtained from non-castrated physiological control rats $(n=8)$, castrated rats implanted with implant PBS $(n=6)$, implant HPCD $(n=7)$, implant testosterone powder $(n=9)$, nDS 1:2 TES: $\operatorname{HPCD}(n=8)$ and degradable pellet $(n=6)$ as positive controls. b) Plasma LH and c) FSH levels obtained from all animal groups. The $\gamma$-axis is truncated and scaled for image clarity. 
median age of the male population rises. At present, there is a need to develop modes of TRT that deliver testosterone in a sustained, constant, and long-term fashion. Here, we demonstrate that the implantable nanochannel system platform is capable of releasing bioactive testosterone i) in a continuous and sustained manner, ii) at a rate feasible for clinical application, and iii) in a manner scalable for clinical use with durations up to 12 months.

In this study, we faced two main experimental challenges, testosterone solubility, and retention of bioactivity. The poor aqueous solubility of testosterone is due to planar polarity of its structure with the presence of a polar -OH moiety and nonpolar moieties at the opposite ends of the cyclopentanoperhydrophenanthrene ring (Figure 1a).

When released in water, testosterone exhibits a constant and sustained release regardless of the channel size (Figure 1c). Such release kinetics is not mediated by constrained diffusion but driven by a constant transmembrane concentration gradient. Testosterone concentration in the implant is maintained at the solubility limit by slowly solubilizing testosterone powder. Unfortunately, the achieved doses with testosterone in this formulation are too low to be clinically relevant; therefore, we examined HPCD as a solubilizer. HPCD is composed of seven 2-hydroxypropyl glucose units joined together to create a lipophilic cavity. The "A" and "D" rings of a testosterone molecule (Figure 1a) enter into the lipophilic cavities of two CD molecules forming a 1:2 stoichiometric inclusion complex. ${ }^{[16]}$ According to our results, such complex involving one testosterone and two CD molecules, greatly enhance testosterone solubility while maintain structural stability. We postulated that at the less optimal 1:1 ratio, three types of molecules or complexes are present: i) 1:2 TES:HPCD, ii) 1:1 TES:HPCD, and iii) uncomplexed testosterone. From this mixture, 1:2 TES:HPCD is rapidly released and depleted, displaying an initial high testosterone delivery rate (Figure $2 \mathrm{~g}$ ). The release of the less soluble 1:1 TES:HPCD and uncomplexed testosterone follows, resulting in a substantial drop in release profile eventually converging to release rates obtained with pure testosterone powder (Figure 1c). The hydrodynamic radius $\left(r_{\mathrm{h}}\right)$ of the 1:2 TES:HPCD complex can be estimated equal to $1.5 \mathrm{~nm}$, by considering a $\beta$-cyclodextrin $r_{\mathrm{h}}$ of $0.77 \mathrm{~nm} \cdot{ }^{[17]}$ Achieving constant release from $5 \mathrm{~nm}$ nanochannels is therefore consistent with our previous findings that a nanochannel size of approximately three times the $r_{\mathrm{h}}$ of the diffusing analyte is needed to obtain a confinement effect and constant delivery.

To examine the feasibility of nanochannel system-based TRT, we analyzed the release and bioactivity of 1:2 TES:HPCD in vivo in castrated rats. Unlike degradable pellets, the implantable nanochannel system releasing TES:HPCD achieved and maintained serum testosterone levels on par with non-castrated, physiological controls. Examination of LH and FSH levels indicated that the complexed testosterone released through the implant still maintained physiological activity in vivo. Testosterone delivery via the nanochannel implant system was therefore successfully demonstrated for a period of $35 \mathrm{~d}$. However the system could be easily scaled up for clinical use. Number of nanochannels on the membrane can be increased to achieve the desired release rate still maintaining reservoir size suitable to contain sufficient amount of lyophilized drug for up to 12 months.

\section{Conclusion}

Testosterone delivery via the nanochannel implant system mitigates hormone peaks and troughs associated with other TRT modalities and could become an attractive alternative strategy for the long-term treatment of male hypogonadism.

\section{Experimental Section}

Materials: Testosterone, deuterated isotope $(98.4 \%)$ was obtained from Sigma-Aldrich; ammonium acetate, acetonitrile, methanol from Fisher Scientific; 2-hydroxypropyl $\beta$-cyclodextrin from Acros organics. Commercially available degradable pellets were used containing $98 \%$ wt testosterone, and $2 \%_{\mathrm{wt}}$ stearic acid and polyvinylpyrrolidone.

In Vitro Release from the Implantable Nanochannel System: Nanochannel membrane and assembly are described elsewhere. ${ }^{[5]}$ Briefly, membranes were attached to Gr2 titanium reservoirs (NanoMedical Systems) with medical-grade epoxy (OG116-31, Epoxy Technology) and cured by UV light. Testosterone $(1.5-2.0 \mathrm{mg}$ ) was loaded with $\approx 845 \mu \mathrm{L}$ of MilliQ water. Implants were placed in $40 \mathrm{~mL}$ of MilliQ water at $37^{\circ} \mathrm{C}$ with stirring $(n=3) .200 \mu \mathrm{L}$ samples were collected every $24 \mathrm{~h}$. Fresh MilliQ water was replaced at each sampling time. Samples were diluted with methanol $(1: 1 \mathrm{v} / \mathrm{v})$, stored at $4{ }^{\circ} \mathrm{C}$, and their testosterone content measured by HPLC at $242 \mathrm{~nm}$. Additional $800 \mu \mathrm{L}$ was collected for bioactivity analysis and measurement of HPCD.

HPLC Analysis: Testosterone was measured using Hitachi Chromaster HPLC. The separation was performed using a HyperSil $\mathrm{C}_{18}$ column (Thermo scientific) attached to Zorbax Eclipse plus $\mathrm{C}_{18}$ guard column $(4.6 \mathrm{~mm} \times 12.5 \mathrm{~mm})$ at $23{ }^{\circ} \mathrm{C}$. Methanol and water $(80: 20 \mathrm{v} / \mathrm{v})$ at isocratic flow rate of $1 \mathrm{~mL} \mathrm{~min}{ }^{-1}$ were used for elution.

Bioactivity Analysis: To determine the bioactivity of TES released from nanochannel membranes in vitro, LNCaP cells were transduced with lentiviral particles using the Cignal Androgen Receptor Reporter (LUC) kit from Qiagen. The transduced cells, LNCaP/LUC, produced light when exposed to testosterone by expressing the firefly luciferase gene, which was then measured with a luminometer (Synergy H4 Hybrid Multi-Mode Microplate Reader, BioTek) to determine the concentration of testosterone in the samples. For the bioactivity experiments, LNCaP/ LUC cells were exposed to different concentrations of testosterone to obtain the expected "S-shaped" standard curve, which was then used to calculate testosterone concentration in the samples from the in vitro release studies. LNCaP/LUC cells were plated in RPMI medium $+10 \%$ FBS $+1 \%$ glutamax $+1 \%$ penicillin/streptomycin $(P / S)$ and grown until cells reached $80 \%-90 \%$ confluency. Cells were then transferred to 96 -well plates (CellBind, Corning) at a density of 20000 cells well $^{-1}$ in $200 \mu \mathrm{L}$ of RPMI no phenol red medium $+5 \%$ charcoal stripped FBS $(\mathrm{CS}-\mathrm{FBS})+1 \%$ glutamax $+1 \% \mathrm{P} / \mathrm{S}$. Ten concentrations of testosterone in the range 10-300 $000 \times 10^{-12} \mathrm{M}$, and wells containing cells and no testosterone (control) were used to determine the testosterone standard curve. Samples from the in vitro release studies were filter sterilized and analyzed by HPLC to determine testosterone concentration before they were added to experimental plates containing the cells. Each sample was added at a concentration of $2000-3000 \times 10^{-12} \mathrm{M}$. Six repetitions per treatment were tested in each experiment. After $24 \mathrm{~h}$, cell lysates were prepared following the protocol for mammalian cells described in the Luciferase Assay System from Promega. Luminescence produced by the cells was measured with a luminometer.

Phase Solubility: Phase solubility testing was performed as described elsewhere..$^{[9]}$ Testosterone powder $(2-3 \mathrm{mg})$ was incubated at 25 and $37^{\circ} \mathrm{C}$ in $2 \mathrm{~mL}$ aqueous HPCD solutions of different concentrations. After $24 \mathrm{~h}$, filtered samples were diluted with methanol $(1: 1)$ and measured by HPLC.

Preparation of TES:HPCD Complexes: A qualitative analysis of TESHPCD interaction was performed with molar rations $1: 1$ and $1: 2$ by LeadIT software. ${ }^{[10]}$ The structure of $\beta$-cyclodextrine was obtained from PDB 1P2G, ${ }^{[1]}$ while the structure of testosterone was acquired from 
DrugBank. ${ }^{[12]}$ The double HPCD was created by mirroring a HPCD molecule and translating its copy at a distance ensuring van der Waals interactions. The cavity created by both HPCD molecules was used to evaluate testosterone affinity for a 1:2 TES:HPCD complex. Interactions were driven by enthalpy and entropy approach. For the experimental analysis, TES:HPCD complexes were prepared in the molar ratios of 1:0.4, 1:1, 1:2, and 1:4 by dissolving testosterone and HPCD in methanol, sonicating the solution, and evaporating the solvent at $60{ }^{\circ} \mathrm{C}$ and $50 \mathrm{rpm}$ (Rotavapor R-210, Buchi).

Measurement of HPCD: HPCD concentration was determined by the reduction in UV absorbance of phenolphthalein. ${ }^{[13]} 1 \mathrm{~mL}$ of $3 \times 10^{-3} \mathrm{M}$ ethanolic phenolphthalein solution and $0.5 \mathrm{~mL}$ of $1 \mathrm{~N}$ aqueous $\mathrm{NaOH}$ solution were added to $18.5 \mathrm{~mL}$ of $170 \times 10^{-3} \mathrm{M} \mathrm{Na}_{2} \mathrm{HCO}_{3}$ to prepare working reagent. The samples were mixed with working reagent at 1:1 $\mathrm{v} / \mathrm{v}$ and the absorbance measured at $554 \mathrm{~nm}$.

Analysis of Residual Testosterone: The content of recovered capsules was dissolved in $10 \mathrm{~mL}$ methanol, centrifuged $(5000 \mathrm{~g}, 5 \mathrm{~min}$ ), further diluted 1:10 v/v with methanol and analyzed by HPLC. For HPCD measurement, $100 \mu \mathrm{L}$ of the supernatant was diluted to $1 \mathrm{~mL}$ with MilliQ water. Average daily release $=$ (amount loaded-residual amount) $/$ days.

In Vivo Study: Male castrated and non-castrated Sprague-Dawley (SD) rats (Harlan Laboratories) were treated per the approved protocol (AUP0411-0018) from the IACUC of The Houston Methodist Research Institute. Before implantation, blood baseline values were established. Castrated animals were randomized into 5 groups $(n=6-9)$ : i) Implant HPCD, ii) Implant PBS, iii) Degradable pellet, iv) Implant TES powder PBS, and v) Implant 1:2 TES:HPCD PBS. Nanochannel implants and degradable pellets were subcutaneously inserted in anesthetized animals $3 \%$ isoflurane). Standard care, including analgesic post-suturing, bupivacaine (4-5 $\mathrm{mg} \mathrm{kg}^{-1}$ ) was provided. Carprofen $\left(5 \mathrm{mg} \mathrm{kg}^{-1}\right.$, subcutaneous) was administered after 24 and $48 \mathrm{~h}$ post-surgery. Blood samples were collected from the saphenous vein. Plasma was separated at $3000 \mathrm{~g}$.

Plasma Testosterone, LH, and FHS Analysis: $20 \mu \mathrm{L}$ of EDTA-treated plasma was mixed with $60 \mu \mathrm{L}$ of the internal D3 standard, mixed by vortexing for $1 \mathrm{~min}$, incubated for $15 \mathrm{~min}$ at room temperature followed by centrifugation at $14000 \mathrm{~g}$ for $10 \mathrm{~min}$ to separate precipitated proteins. Supernatant was vacuum dried (Speedvac, ThermoScientific) and resuspended in $40 \mu \mathrm{L}$ of $60 \%: 40 \%$ methanol:water and $0.1 \%$ ammonium acetate with average recovery $\approx 75 \%$. Separation was carried out on a Acquity UPLC system (Waters Corp.) using an UPLC BEH C-18 column (2.1 $\mathrm{mm} \times 50 \mathrm{~mm} ; 1.7 \mu \mathrm{m}$ particle). Each sample was analyzed in duplicate. Average testosterone recovery from plasma, as measured by internal standard D3, was approximately $75 \%$ for the extraction method. Mass spectrometry was performed on a Waters Xevo TQ tandem quadrupole mass spectrometer operated in the SRM/MRM mode under positive ion electrospray conditions. Data acquisition was done by MassLynx v4.1 and sample quantification by TargetLynx v4.1 (Waters Corp.) (see details in Supporting Information Section 1). Analysis of LH and FSH plasma levels was performed at the Ligand Core (University of Virginia, VA, USA) with the Millipore Multiplex Endocrine Assay.

\section{Supporting Information}

Supporting Information is available from the Wiley Online Library or from the author.

\section{Acknowledgements}

A.G., M.K., and G.P. are co-senior authors of this work. The authors express their gratitude to Sharath Hosali for the microfabrication of the nanochannel membranes, Alma Zecevic for the finalization of the manuscript and the HMRI Proteomic Core for plasma testosterone quantification. The study was supported with funds from the Houston Methodist Research Institute and the CPRIT Innovative thinking fellowship. The authors M.F., R.G., L.H, M.K., G.P., and A.G. disclose a financial interest in NanoMedical Systems, Inc. M.K. also discloses a financial interest in Merck, Lilly, Auxilium and Meda. All other authors disclose no competing financial interest.

Received: June 23, 2014

Revised: July 21, 2014

Published online: October 1, 2014

[1] a) A. A. Yassin, M. Haffejee, Clin. Interv. Aging 2007, 2, 577; b) A. S. Dobs, A. W. Meikle, S. Arver, S. W. Sanders, K. E. Caramelli, N. A. Mazer, J. Clin. Endocrinol. Metab. 1999, 84, 3469; c) M. J. Cukierski, P. A. Johnson, J. C. Beck, Int. J. Toxicol. 2001, 20, 369.

[2] a) A. K. Bang, N. Jorgensen, E. Rajpert-De Meyts, A. Juul, Front Endocrinol. 2013, 4, 94; b) H. W. Daniell, R. Lentz, N. A. Mazer, J. Pain 2006, 7, 200; c) A. R. McCullough, M. Khera, I. Goldstein, W. J. Hellstrom, A. Morgentaler, L. A. Levine, J. Sex Med. 2012, 9, 594; d) E. D. Grober, M. Khera, S. D. Soni, M. G. Espinoza, L. I. Lipshultz, Int. J. Impot. Res. 2008, 20, 213; e) M. Marberger, A. V. Kaisary, N. D. Shore, G. S. Karlin, C. Savulsky, R. Mis, C. Leuratti, J. R. Germa, Clin. Ther. 2010, 32, 744.

[3] T. Mulligan, M. F. Frick, Q. C. Zuraw, A. Stemhagen, C. McWhirter, Int. J. Clin. Pract. 2006, 60, 762.

[4] R. Sih, J. E. Morley, F. E. Kaiser, H. M. Perry, 3rd, P. Patrick, C. Ross, J. Clin. Endocrinol. Metab. 1997, 82, 1661.

[5] S. Ferrati, D. Fine, J. You, E. De Rosa, L. Hudson, E. Zabre, S. Hosali, L. Zhang, C. Hickman, S. Bansal, A. Cordero-Reyes, T. Geninatti, J. Sih, R. Goodall, G. Palapattu, M. Kloc, R. Ghobrial, M. Ferrari, A. Grattoni, J. Controlled Release 2013, 172, 1011.

[6] a) J. Sih, S. S. Bansal, S. Filipini, S. Ferrati, K. Raghuwansi, E. Zabre, E. Nicolov, D. Fine, M. Ferrari, G. Palapattu, A. Grattoni, Anal. Bioanal. Chem. 2013, 405, 1547; b) C. Celia, S. Ferrati, S. Bansal, A. L. van de Ven, B. Ruozi, E. Zabre, S. Hosali, D. Paolino, M. G. Sarpietro, D. Fine, M. Fresta, M. Ferrari, A. Grattoni, Adv. Healthcare Mater. 2014, 3, 230.

[7] a) D. Fine, A. Grattoni, E. Zabre, F. Hussein, M. Ferrari, X. Liu, Lab Chip 2011, 11, 2526; b) A. Grattoni, H. Shen, D. Fine, A. Ziemys, J. S. Gill, L. Hudson, S. Hosali, R. Goodall, X. Liu, M. Ferrari, Pharm. Res. 2011, 28, 292.

[8] a) A. Grattoni, J. Gill, E. Zabre, D. Fine, F. Hussain, M. Ferrari, Anal. Chem. 2011, 83, 3096; b) A. Pimpinelli, M. Ferrari, A. Grattoni, Appl. Phys. Lett. 2013, 103, 113104; c) A. Ziemys, M. Kojic, M. Milosevic, N. Kojic, F. Hussain, M. Ferrari, A. Grattoni, J. Comput. Phys. 2011, 230, 5722 .

[9] N. Nasongkla, A. F. Wiedmann, A. Bruening, M. Beman, D. Ray, W. G. Bornmann, D. A. Boothman, J. Gao, Pharm. Res. 2003, 20, 1626.

[10] C. Chen, J. Skog, C. H. Hsu, R. T. Lessard, L. Balaj, T. Wurdinger, B. S. Carter, X. O. Breakefield, M. Toner, D. Irimia, Lab Chip 2010 10, 505.

[11] N. Pinotsis, D. D. Leonidas, E. D. Chrysina, N. G. Oikonomakos, I. M. Mavridis, Protein Sci. 2003, 12, 1914.

[12] C. Knox, V. Law, T. Jewison, P. Liu, S. Ly, A. Frolkis, A. Pon, K. Banco, C. Mak, V. Neveu, Y. Djoumbou, R. Eisner, A. C. Guo, D. S. Wishart, Nucleic Acids Res. 2011, 39, D1035.

[13] S. C. Szathmary, J. Chromatogr. 1989, 487, 99.

[14] A. Grattoni, D. Fine, E. Zabre, A. Ziemys, J. Gill, Y. Mackeyev, M. A. Cheney, D. C. Danila, S. Hosali, L. J. Wilson, F. Hussain, M. Ferrari, ACS Nano 2011, 5, 9382.

[15] S. Bhasin, G. R. Cunningham, F. J. Hayes, A. M. Matsumoto, P. J. Snyder, R. S. Swerdloff, V. M. Montori, J. Clin. Endocrinol. Metab. 2010, 95, 2536.

[16] W. Cai, T. Sun, P. Liu, C. Chipot, X. Shao, J. Phys. Chem. B 2009 113,7836

[17] G. M. Pavlov, E. V. Korneeva, N. A. Smolina, U. S. Schubert, Eur. Biophys. J. 2010, 39, 371. 